\title{
Stress of COVID-19 and mental health outcomes in Palestine: the mediating role of well-being and resilience
}

\begin{abstract}
BACKGROUND
We tested the association between the stress of COVID-19 and mental distress manifested by anxiety, depression, and stress, and whether well-being and resilience mediate the relationship between the two variables in a population of Palestinian adults. Namely, we hypothesized that stress of COVID-19 will be positively associated with mental health problems (anxiety, depression, and stress); well-being and resilience will be negatively associated with mental health problems (anxiety, depression, and stress); and well-being and resilience will mediate the association between the stress of COVID-19 and mental health problems (anxiety, depression, and stress).
\end{abstract}

\section{PARTICIPANTS AND PROCEDURE}

Depression, Anxiety and Stress Scale-21, the Resilience Scale for Adults, the WHO-5 Well-Being Index, and the COVID-19 Stress Scales were administered to 860 participants via online survey. Structural equation modelling (SEM) was applied to address the study hypothesis.

\begin{abstract}
RESULTS
Participants recorded a mild level of stress of COVID-19 and high scores for resilience and well-being. A conceptual model depicting COVID-19 stress as a predictor, mental distress as an outcome variable, and resilience and subjective well-being as mediators was confirmed.
\end{abstract}

\section{CONCLUSIONS}

Palestinians are exposed to mental distress in an environment characterized by ongoing stressors. Conversely, the capability of Palestinians to conserve good levels of wellbeing and resilience protects them from mental distress even in the wake of the COVID-19 outbreak. On the other hand, COVID-19 can be considered a new critical event demanding and diminishing the people's resiliency recourses in Palestine. Implications for clinical practice and research are discussed.

\section{KEY WORDS}

COVID-19; mental distress; resilience; subjective well-being

ORGANizATION - 1: University of Milano-Bicocca, Milan, Italy · 2: An-Najah National University, Nablus, Occupied Palestinian Territories

AUthors' Contributions - A: Study design - B: Data collection - C: Statistical analysis - D: Data interpretation .

E: Manuscript preparation · F: Literature search · G: Funds collection

CORRESPONDING AUthor - Prof. Guido Veronese, Department of Human Sciences and Education, University of Milano-Bicocca, Piazza dell' Ateneo Nuovo 1, 20126 Milan, Italy, e-mail: guido.veronese@unimib.it

to Cite this ARTICLE - Veronese, G., Mahamid, F., Bdier, D., \& Pancake, R. (2021). Stress of COVID-19 and mental health outcomes in Palestine: the mediating role of well-being and resilience. Health Psychology Report, 9(4), 398-410.

https://doi.org/10.5114/hpr.2021.104490

RECEIVED 10.12.2020 • REVIEWED 13.01.2021 · ACCEPTED 29.01.2021 · PUBLISHED 15.03.2021 


\section{BACKGROUND}

As the confirmed cases of coronavirus (COVID-19) approached 200,000 individuals across 160 countries, the World Health Organization (WHO) declared COVID-19 a pandemic (Spinelli \& Pellino, 2020). COVID-19 disease is a pneumonia pandemic, caused by severe acute respiratory syndrome coronavirus 2 (SARS-CoV-2) (Dashraath et al., 2020). Patients with COVID-19 disease typically present with fever, shortness of breath and cough (Poyiadji et al., 2020).

COVID-19 is characterized as being highly contagious and rapidly spreading. At the time of writing (July 26, 2020), there were 15,785,641 confirmed cases of COVID-19, including 640,016 deaths, globally (WHO, 2020a). From these, there were 12,795 confirmed cases with 78 deaths documented in the occupied Palestinian territory (WHO, 2020b).

As COVID-19 was found to be quickly and easily transmitted, individuals experienced an increase in stress and worry about themselves and their families contracting the COVID-19 disease (Wang et al., 2020a, b). Additionally, isolation and quarantine strategies, overloaded misinformation, stigma, ambiguity of treatment possibilities and economic hardship (Burtscher et al., 2020; Dong \& Zheng, 2020; Gao et al., 2020; Islam et al., 2020) caused individuals around the globe to experience higher levels of chronic stress (Torales et al., 2020). Individuals found themselves unable to cope as the stressor (i.e. COVID-19 disease) mismatched their internal resources (Aldwin, 2007).

As stressful situations can increase common mental health problems such as anxiety and depression (Ahmed et al., 2020), the stress of COVID-19 impacted the psychological distresses among individuals. For example, stress related to COVID-19 was found to be associated with anxiety among people who quarantined at home for 14 days during the COVID-19 epidemic in central China (Xiao et al., 2020). Islam et al. (2020) found that COVID-19-related stress resulted in shortened sleep, a short temper, and chaos among families in Bangladesh. Stress related to COVID-19 predicted anxiety, depression, and stress among individuals who had been through a strict quarantine since March 2020 and were now experiencing a more relaxed lockdown in Philippines (Montano \& Acebes, 2020). Financial distress was found to be associated with loneliness, stress, anxiety, depression, and suicidal behavior among individuals from the UK, the USA, and Canada (Stevenson \& Wakefield, 2020). Lastly, results show that xenophobia is a common product of pandemics, harming the overall well-being of Indians (Ahuja et al., 2020).

Palestinian individuals are expected to suffer from several mental health problems related to the stress of COVID-19 as they already face several stressors on a daily basis, including political, economic, and social stressors such as gender discrimination, the inabil- ity to move freely between the Palestinian territories because of the Israeli occupation restrictions and lack of recreational facilities. Economic stressors are characterized by the scarcity of employment opportunities and high rates of poverty, while the primary political stressor is characterized by the harsh consequences of Israeli militarization (Mahamid \& Berte, 2019, 2020). Another potential stressor is the large number of Palestinians who work in Israeli settlements which are identified as highly infected areas, increasing the workers' exposure to the virus and the potential of infecting their families and neighbors (Mahamid et al., 2020a).

In respect to this study, the stress of COVID-19 is predicted to be associated with different mental health problems among Palestinians. Recent studies show that the outbreak of COVID-19 and fear of COVID-19 are positively associated with high rates of multiple psychological impairments such as anxiety, stress and depression among Palestinians (Mahamid \& Bdier, 2020a, b; Mahamid et al., 2020b).

Conversely, there are several psychological factors that may mitigate mental health problems related to the stress of the COVID-19 outbreak. Subjective well-being, which has been best defined as the extent to which a person is satisfied, happy and enjoys life (Agbaria \& Natur, 2018), can serve as an effective buffer to COVID-related stressors. Subjective well-being is considered a protective factor in which individuals with this trait are more capable of coping with stressful conditions (Freire et al., 2016) and less vulnerable to mental distress (Layous et al., 2014).

Individuals with high rates of well-being were found to be happier than their counterparts during COVID-19 (Yang \& Ma, 2020). Well-being was found to mediate the relationship between the fear of COVID-19 and psychological distress among psychosocial service providers in Palestine (Mahamid \& Bdier, 2020b). In Poland, research results indicated that stronger basic hope and higher levels of meaning in life and life satisfaction correlated with lower state anxiety and lower COVID-19 stress among individuals (Trzebiński et al., 2020). Additionally, optimism was found to be a protective factor in mitigating the impact of stress on depressive symptoms among Turkish university students during COVID-19 (Arslan \& Yildirim, 2020a).

Furthermore, resilience can be an important factor in protecting against psychological distress. For the purposes of this study, resilience refers to competencies to cope successfully with stressful experiences, to control irrational thoughts related to the stressor, and to successfully overcome stressors despite the magnitude of challenges related to it (Zolkoski \& Bullock, 2012).

A recent study showed that resilience mediated the relationship between hope, psychological functioning, and subjective well-being among Turkish
COVID-19 stress, mental distress, resilience, and well-being 
Guido Veronese, Fayez Mahamid, Dana Bdier, Rachel Pancake adults during the early stage of the COVID-19 outbreak (Yildirim \& Arslan, 2020). In the United States, resilience was found to be negatively associated with depression and anxiety symptoms among young adults during the COVID-19 pandemic (Liu et al., 2020). Similar findings were obtained by Kavčič et al. (2021) as they found resilience to be a crucial factor in promoting good psychological functioning during the COVID-19 pandemic among Slovene adults.

In addition, Arslan and Yildirim (2020b) investigated the mediating effect of affect balance and resilience on the association between meaningful living and psychological impairments among Turkish young adults in the context of the pandemic. The findings showed that meaningful living had a positive predictive effect on resilience and positive effects, as well as a negative association on psychological distress and negative affects. In contrast, resilience and affect balance also mediated the effect of meaningful living on psychological health of the participants in this research.

Palestinian people are expected to have an increased risk of psychological burdens such as depression, anxiety, and stress during the COVID-19 pandemic due to the political, economic, and social instability of their region. However, well-being and resilience are predicted to be protective factors for psychological functioning related to COVID-19 stress (Veronese et al., 2020b). As demonstrated by a previous study, Palestinians attempt to build their resilience and well-being through the application of different coping strategies and survival skills to tolerate and manage their traumatic reality (Veronese et al., 2020b). In a comparative study between Palestinian and Jordanian adults, levels of well-being, quality of life and stress among Palestinians were healthier than in their Jordanian counterpart. The authors concluded that Palestinian adults developed a culture of resilience (Asi et al., 2018). Moreover, resilience is found to be positively correlated with positive affects among Palestinians (Kteily-Hawa et al., 2020).

Theoretically speaking, we refer to the WHO definition of mental health as a "state of well-being in which the individual realizes his or her own abilities, copes with the normal stresses of life, works productively and fruitfully, and makes a contribution to his or her community" (WHO, 2001, p. 1). Constructs of subjective well-being (SWB) and resilience unite to comprise the WHO's definition of psychological functioning. If SWB is seen as the individual's capacity to conserve a sense of endurance and competence in the wake of adverse conditions, then it might be considered a protective factor that ensures people's positive functioning and mental health during the COVID-19 outbreak (Yildirim \& Arslan, 2020). In addition, resilience was found to have a positive impact on mental health and to be associated with subjective well-being. Thus, both SWB and resilience might be studied as mediators between COVID-19 stress and mental distress.

Accordingly, the current exploratory pilot study examines the relationship between stress of COVID-19 and mental health impairments operationalized in term of anxiety, depression, and stress, and whether well-being and resilience mediate the relationship between these two variables. Based on prior research, three hypotheses were defined for this study: the stress of COVID-19 will be positively associated with mental health problems (anxiety, depression, and stress) (H1); well-being and resilience will be negatively associated with mental health problems (anxiety, depression, and stress) (H2); well-being and resilience will mediate the association between the stress of COVID-19 and mental health problems (anxiety, depression, and stress) (H3).

\section{PARTICIPANTS AND PROCEDURE}

\section{PARTICIPANTS}

Participants were recruited from online advertisements, e-mail campaigns and social media. There were 860 participants: 189 males and 671 females. The majority (57.2\%) of participants were from cities, $33.7 \%$ were from villages and $9.1 \%$ were from Palestinian refugee camps. A sum of $85 \%$ of participants held an academic degree and $15.0 \%$ did not. Inclusion in the study required participants to be Palestinian, native Arabic speakers, and living in the West Bank of the occupied Palestinian territory during the spread of COVID-19.

\section{MEASURES}

Following standard methodological recommendations for the development of our questionnaires (Hambleton et al., 2004), all items were translated and back translated from the original English version to Arabic and pilot-tested by a panel of ten Arab professionals recognized as experts in psychology, counseling, and social work. These professionals evaluated the clarity and relevance of the questions and translation. After completion of the translated draft, the questionnaires were back-translated into English by an independent expert English editor. The translated version was then pilot tested among 80 participants and further refined for clarity according to their comments.

Depression, Anxiety and Stress Scale (DASS-21). The DASS-21 is a 21-item self-reported questionnaire designed by Lovibond and Lovibond (1995) to measure the severity of a range of symptoms common in both depression and anxiety. In completing the DASS21, the individual is required to indicate the presence of a symptom over the previous week. Each 
item is scored from 0 (did not apply to me at all over the last week) to 3 (applied to me very much or most of the time over the past week). The essential function of the DASS-21 is to assess the severity of the core symptoms of depression, anxiety, and stress.

The WHO-5 Well-Being Index. The 5-item World Health Organization Well-Being Index (WHO-5) is a short and generic global rating scale measuring subjective well-being. Because the WHO considers positive well-being to be another term for mental health, the WHO-5 only contains positively phrased items. These items are: (1) "I have felt cheerful and in good spirits", (2) "I have felt calm and relaxed", (3) "I have felt active and vigorous", (4) "I woke up feeling fresh and rested", and (5) "My daily life has been filled with things that interest me". The respondent is asked to rate how well each of the 5 statements applies to him or her when considering the last 14 days. Each of the five items is scored from 5 (all the time) to 0 (none of the time). The raw score therefore theoretically ranges from 0 (absence of well-being) to 25 (maximal well-being) (Staehr, 1998).

The COVID Stress Scales (CSS). The CSS were constructed by Taylor et al. (2020) to assess levels of stress related to the COVID-19 outbreak. The scales include six domains: (1) fears about the dangerousness of COVID-19, (2) fears about sources of COVID-19-related contamination (i.e., objects, surfaces), (3) COVID-19 xenophobia (i.e., fears that foreigners are sources of COVID-19), (4) fears about the personal social and economic consequences of COVID-19 (e.g., fears of disruption in the supply chain, fears of looting or rioting), (5) COVID-19-related checking (e.g., checking news media or social media, seeking reassurance from friends or medical professionals), and (6) traumatic stress symptoms related to COVID-19 (e.g., unwanted intrusive thoughts or nightmares relating to COVID-19). Items are rated on a 5-point scale ranging from 0 (not at all) to 4 (extremely).

Resilience Scale for Adults (RSA). The RSA is a 33-item self-report scale prepared by Friborg et al. (2003) for measuring protective resilience factors among adults. The scale comprises five factors labeled: personal competence ("I know if I continue, I will succeed"), social competence ("I can establish friendly relationships easily"), family cohesion ("Even in difficult situations, my family is optimistic"), social resources ("There is always someone who helps me when I'm in need") and structured style ("I sustain my daily rules even in difficult situations"). Respondents rated items using a Likert response format with gradations from 1 (strongly disagree) to 5 (strongly agree).

\section{PROCEDURE}

Research was conducted in August 2020 and targeted Palestinians during a lockdown period in the West
Bank of Palestine due to the spread of COVID-19 pandemic. The sample was recruited online using convenience sampling techniques. All participants were provided with information that enabled them to make informed decisions as to whether they wanted to participate in the research. They were provided with descriptions of the scales and the purpose of the research. Participants who agreed to participate in the research signed an informed consent form. The research was conducted in line with the ethical guidelines of the American Psychological Association (APA, 2010) and the Declaration of Helsinki (2013) and had been approved by the An-Najah National University IRB (Protocol number 16 May).

\section{DATA ANALYSIS}

Structural equation modeling (SEM; Gunzler et al., 2013) was used to test a conceptual model where well-being and resilience were identified as mediators. Furthermore, COVID-19 stress was considered as a predictor and mental distress, operationalized in term of depression, anxiety, and stress, as an outcome variable. We explored statistical distribution of the data for each of the variables. Both kurtosis and skewness values fell inside the recommended cutoffs $[-1,+1]$. Moreover, we calculated Mahalanobis' distance $(p<.001)$ for all scores to detect and omit multivariate outliers: no extreme multivariate values were found. We adopted two fit indexes: absolute and relative. The selected absolute indexes were $\chi^{2}$ and normed $\chi^{2}$ (NC) as a non-statistically significant $\chi^{2}$ value and NC values under 2.0 indicate good fit (Hair et al., 2010). Additionally, root mean square error of approximation (RMSEA), normed fit index (NFI), nonnormed fit index (NNFI), comparative fit index (CFI) and standardized root mean square (SRMR) were calculated. The thresholds for good fit were as follows: RMSEA < .042 and SRMR < .05 (Schermelleh-Engel et al., 2003), NFI > .94 (Marsh et al., 2004), CFI > .94 (Hu \& Bentler, 1999). Lastly, we set a $p$ value at .003. The SEM model (see Figure 1) has been tested using AMOS 25 software for data analysis.

\section{RESULTS}

Descriptive statistics related to the stress of COVID-19, resilience, well-being, depression, anxiety, and stress are given in Table 1 . Overall, it must be noted that the participants scored within a mild degree in socio-economic, traumatic stress, compulsive checking, and anxiety dimensions, with moderate scores in danger, xenophobia, depression, and depression dimensions. Participants recorded a mild level of stress of COVID-19 and high scores for resilience and well-being. All measures satisfied nor-
COVID-19 stress, mental distress, resilience, and well-being 


\section{Figure 1}

Conceptualized effect of COVID-19 stress on anxiety, depression and stress and the mediating role of resilience and well-being

Guido Veronese, Fayez Mahamid, Dana Bdier, Rachel Pancake

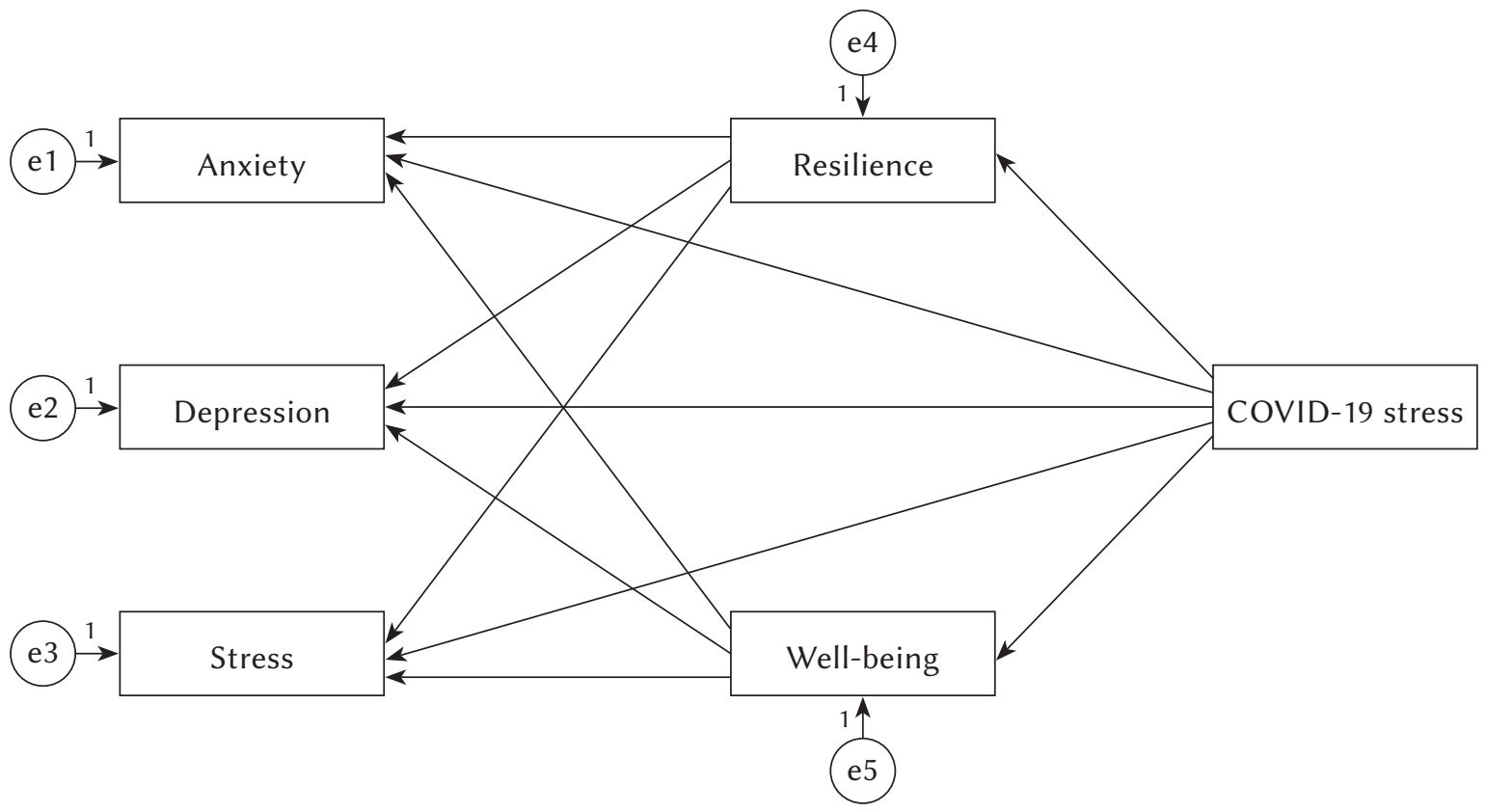

Table 1

Descriptive statistics for research variables $(N=860)$

\begin{tabular}{lcccccccc}
\hline Variable & $M$ & $S D$ & Min & Max & Range & Skewness & Kurtosis & Reliability \\
\hline Danger & 2.29 & 0.92 & .00 & 4.00 & 4.00 & -0.41 & .08 & .82 \\
Socio-economic & 1.34 & 0.87 & .00 & 4.00 & 4.00 & 0.62 & .08 & .89 \\
Xenophobia & 2.50 & 0.69 & .00 & 4.00 & 4.00 & -0.56 & .08 & .88 \\
Contamination & 2.27 & 0.59 & .00 & 4.00 & 4.00 & -0.33 & .08 & .92 \\
Traumatic stress & 0.81 & 0.42 & .00 & 4.00 & 4.00 & 1.42 & .08 & .88 \\
Compulsive checking & 1.46 & 0.90 & .00 & 4.00 & 4.00 & 0.48 & .08 & .82 \\
COVID-19 stress total & 1.74 & 0.75 & .00 & 4.00 & 4.00 & 0.10 & .08 & .95 \\
Anxiety & 1.26 & 0.77 & .00 & 4.00 & 4.00 & 1.60 & .08 & .92 \\
Depression & 2.15 & 0.70 & .00 & 4.00 & 4.00 & 0.76 & .08 & .93 \\
Stress & 2.32 & 0.82 & .00 & 4.00 & 4.00 & 0.58 & .08 & .90 \\
Resilience & 2.89 & 0.69 & .00 & 4.00 & 4.00 & -0.82 & .08 & .93 \\
Well-being & 3.11 & 0.62 & .00 & 5.00 & 5.00 & -0.54 & .08 & .85 \\
\hline
\end{tabular}

mality assumptions. Regarding reliability, all scales displayed high reliability values ranging from .81 (anxiety) to .94 (total COVID-19 stress score).

Results of the correlational analysis are reported in Table 2. Namely, COVID-19 stress total was positively correlated with anxiety $(r=.51, p<.01)$, depression $(r=.47, p<.01)$, and stress $(r=.55, p<.05)$, and negatively correlated with resilience $(r=-.25, p<.01)$, and well-being $(r=-.23, p<.01)$. Moreover, anxiety corre- lated positively with depression $(r=.74, p<.01)$, and stress $(r=.81, p<.01)$ and negatively with resilience $(r=-.24, p<.01)$ and well-being $(r=-.23, p<.01)$. Depression correlated positively with stress $(r=.86$, $p<.01)$, and negatively with resilience $(r=-.41$, $p<.01)$, and well-being $(r=-.43, p<.01)$. Stress correlated negatively with resilience $(r=-.32, p<.01)$, and well-being $(r=-.36, p<.01)$. Finally, resilience correlated positively with well-being $(r=.63, p<.01)$. 


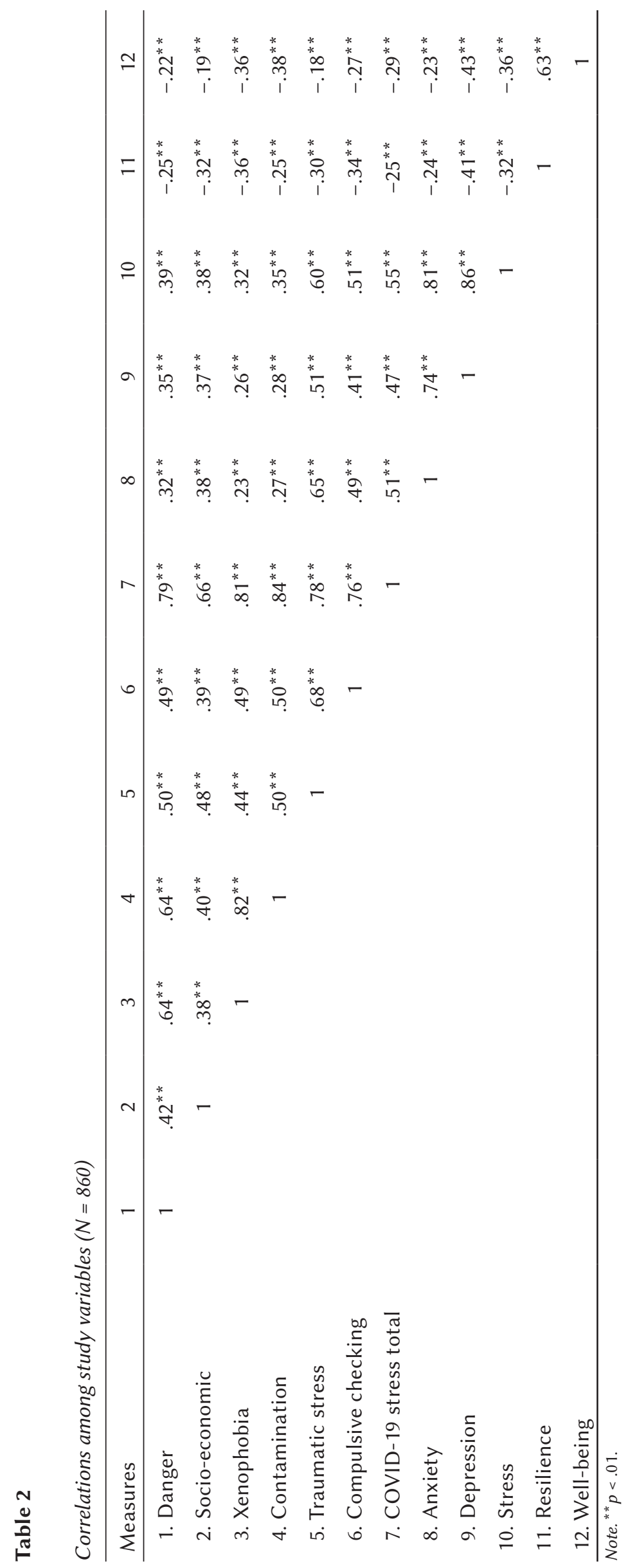




\section{STRUCTURAL EQUATION MODEL (SEM)}

The attained path analysis results are given in Figure 2. The hypothesized model is in Figure 1 with COVID-19 stress as a predictor, resilience and wellbeing as a mediator, and mental health outcomes as a target variable was tested across the sample $(N=860)$. The findings suggested that resilience and well-being mediated the relationship with a good fit for the data $\left(\chi^{2}(4)=35.37, p=.001\right.$, GFI $=.92$, AGFI $=.93$, RMSEA $=.042, \mathrm{NFI}=.94, \mathrm{CFI}=.94)$.

For our study purposes, five main hypotheses were tested with a coefficient and $t$ value. All the $t$ value coefficients are over 1.96; therefore, the three hypotheses could be accepted as shown in Table 3 .

Regarding H1, analysis of the moderating effects showed a negative effect of the COVID-19 stress on mental health outcomes; stress $\left(\beta_{X, Y}=.43, p<.001\right)$,
Guido Veronese, Fayez Mahamid, Dana Bdier, Rachel Pancake

\section{Figure 2}

Structural equation modeling of COVID-19 stress on depression, anxiety and stress and the mediating role of resilience and well-being

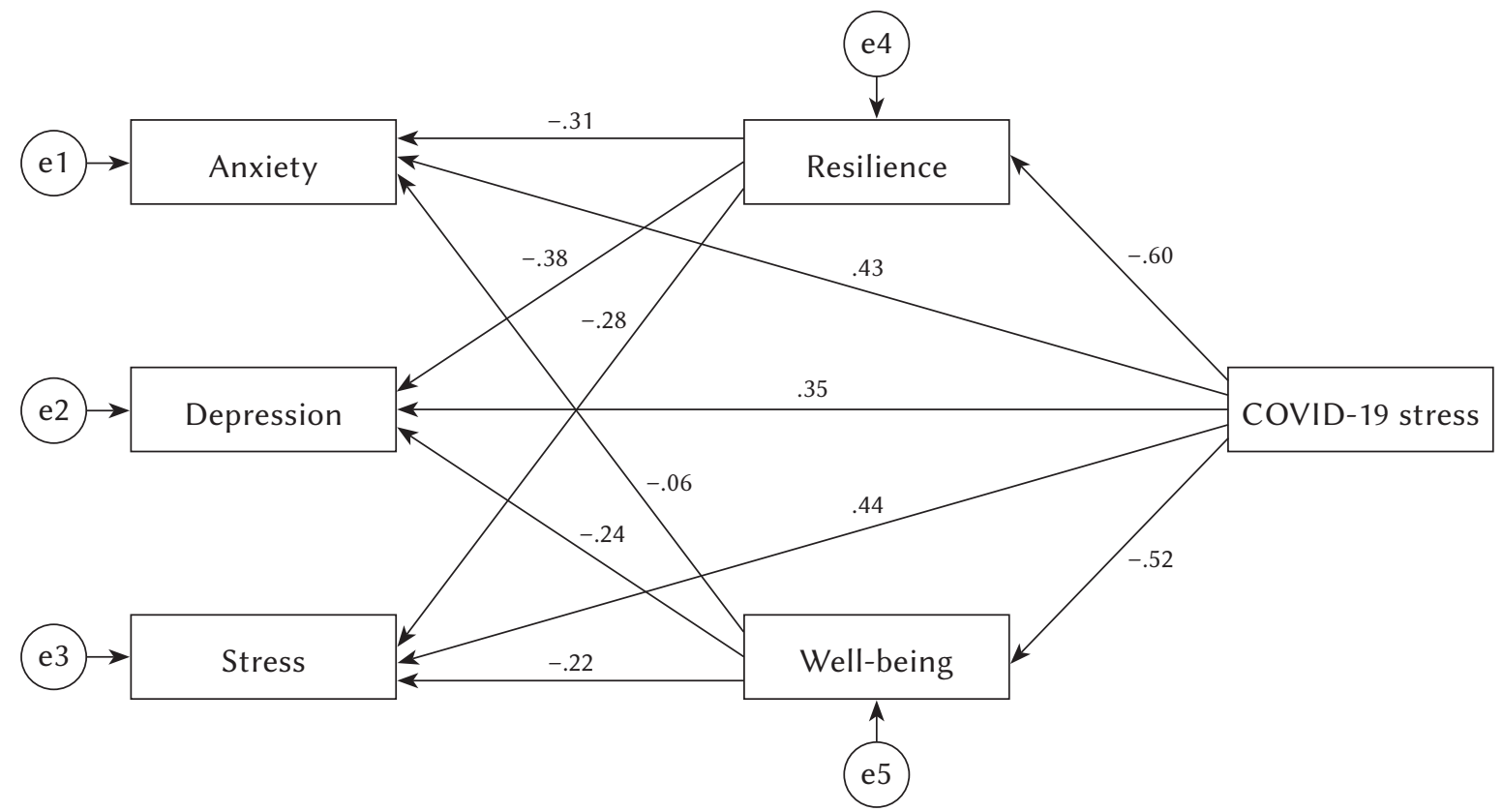

Table 3

Estimates of parameters for the structural equation model $(N=860)$

\begin{tabular}{|c|c|c|c|c|}
\hline Model & Parameter & $\beta$ & $t$ & Hypothesis \\
\hline \multirow[t]{3}{*}{$\mathrm{H} 1$} & COVID-19 stress $\rightarrow$ stress & .44 & $14.62^{* * *}$ & Accepted \\
\hline & COVID-19 stress $\longrightarrow$ depression & .35 & $12.09^{* * *}$ & \\
\hline & COVID-19 stress $\longrightarrow$ anxiety & .43 & $12.59^{* * *}$ & \\
\hline \multirow[t]{3}{*}{$\mathrm{H} 2$} & Well-being $\rightarrow$ stress & -.22 & $-8.93^{* * *}$ & Accepted \\
\hline & Well-being $\rightarrow$ depression & -.25 & $-10.04^{* * *}$ & \\
\hline & Well-being $\longrightarrow$ anxiety & -.06 & -2.10 & \\
\hline \multirow[t]{3}{*}{$\mathrm{H} 3$} & Resilience $\rightarrow$ stress & -.28 & $-10.42^{* * *}$ & Accepted \\
\hline & Resilience $\rightarrow$ depression & -.38 & $-14.49^{* * *}$ & \\
\hline & Resilience $\rightarrow$ anxiety & -.31 & $-10.26^{* * *}$ & \\
\hline $\mathrm{H} 4$ & COVID-19 stress $\rightarrow$ well-being & -.52 & $-17.45^{* * *}$ & Accepted \\
\hline H5 & COVID-19 stress $\rightarrow$ resilience & -.60 & $19.34 * * *$ & Accepted \\
\hline
\end{tabular}

Note. Standardized solutions are reported; ${ }^{* * *} p<.001$. 
depression $\left(\beta_{\mathrm{X}, \mathrm{Y}}=.34, p<.001\right)$, and anxiety $\left(\beta_{\mathrm{X}, \mathrm{Y}}=.42\right.$, $p<.001)$. Analysis of the path between well-being and the mental health outcomes suggested negative effects; stress $\left(\beta_{\mathrm{M}, \mathrm{Y}}=-.24, p<.001\right)$, and depression $\left(\beta_{\mathrm{M}, \mathrm{Y}}=-.24, p<.001\right)$ standardized total direct effect as for $\mathrm{H} 2$. Concerning H3, path analysis between resilience and the mental health outcomes yielded negative effects; stress $\left(\beta_{M, Y}=-.28, p<.001\right)$, depression $\left(\beta_{\mathrm{M}, \mathrm{Y}}=-.37, p<.001\right)$, and anxiety $\left(\beta_{\mathrm{M}, \mathrm{Y}}=-.33, p<.001\right)$.

Finally, concerning the full mediation hypothesis (H4), the model yielded a standardized total effect of well-being $\left(\beta_{\mathrm{X}, \mathrm{M}}=-.52, p<.001\right)$. However, this effect was composed of a statistically significant indirect effect (via well-being, $\beta_{\mathrm{X}, \mathrm{M}, \mathrm{Y}}=-.18, p<.01$ ) and a statistically significant direct effect $\left(\beta_{\mathrm{X}, \mathrm{Y}, \mathrm{M}}=-.33, p<.01\right)$. Concerning $\mathrm{H} 5$, the model also yielded a standardized total effect of resilience $\left(\beta_{\mathrm{X}, \mathrm{M}}=-.56, p<.001\right)$. However, this effect was composed of a statistically significant indirect effect (via resilience, $\beta_{\mathrm{X}, \mathrm{M}, \mathrm{Y}}=-.22$, $p<.01)$ and a statistically significant direct effect $\left(\beta_{\mathrm{X}, \mathrm{Y}, \mathrm{M}}=-.34, p<.01\right)$. This means that the relationship between the stress of COVID-19 and mental health outcomes was fully mediated by well-being and resilience. We also tested the effect of age and gender variables in the model, and no difference was found in perceived stress, mental health and well-being due to these variables.

\section{DISCUSSION}

In this study, we aimed to test subjective well-being and resilience as mediators between COVID-19-related stress and mental health problems, operationalized in term of depression, anxiety, and stress. We tested three hypotheses and drew a conceptual model depicting COVID-19 stress as a predictor, mental health as an outcome variable, and resilience and subjective well-being as connectors. All our hypotheses were confirmed by SEM analysis.

First, we found a direct and positive association between COVID-19 and anxiety, depression, and stress. It means that COVID-19 related stress seems to contribute to increasing the preexisting risks for mental health, revealing its role as a potential activator of mental distress (H1) (Wang et al., 2020a, b; Pfefferbaum \& North, 2020). If we acknowledge the ongoing stressors the population endures because of the Israeli military occupation, COVID-19 related stress could be considered as a critical risk factor undermining the mental health of Palestinians living in the West Bank. The prevalence of mental distress in the occupied Palestinian territory is relevant. Adding COVID-19 stress as an additional determinant for mental health deterioration could put the population at risk for a further decline in their quality of life (Giacaman et al., 2011). Furthermore, the more well-being and resilience decline in our participants, the more mental distress might rise, though the full extent of its impact on mental health is undermined. In essence, a decrease in resources both in terms of subjective well-being and personal, social, and contextual coping strategies is associated with increased levels of depression, anxiety, and stress (H2). The more the stress due to COVID-19 erodes sources of well-being and resilience, the more Palestinians could experience higher mental distress in an environment characterized by stratified and chronic stressors. Conversely, the capability of Palestinians to conserve good levels of well-being and resilience could protect them from mental distress even in the wake of the COVID-19 outbreak (Arslan \& Yildirim, 2020a, b; Veronese et al., 2017, 2020a). In fact, both resilience and subjective well-being displayed a mediating role between COVID-19 stress and mental distress (H3).

According to our results, COVID-19 stress could be an additional critical stressor that might undermine sources of functioning (subjective well-being and resilience) exposing Palestinians to psychological burdens and putting them at a higher risk for mental distress. Social distancing, economic consequences due to the pandemic, an increased sense of isolation and restrictions on travel and movement during the outbreak contribute to weakening an already fragile balance between skills of survival and environmental constraints, exposing people more and more to the consequences of the occupation and ongoing political instability (Hammoudeh et al., 2020).

The mental health of Palestinians was already compromised when the pandemic reached Palestine due to the political context. Since the fall of the West Bank, East Jerusalem and the Gaza Strip in 1967, Palestinians have endured chronic exposure to political violence, oppression, subjugation, and a lack of freedom under Israeli military rule. Generations of Palestinians have suffered human rights violations including: land confiscation, displacement, Israeli army control over the movement of people and goods both domestically and internationally, death, injury, disability, imprisonment and injustice.

As Palestinians who work in Israel returned home for the holidays, cases of COVID-19 began to rise in Palestine. As Israel became the largest source of virus spread in the West (Ghandour et al., 2020), the capacity for Palestinian health care providers to contain the spread of COVID-19 was limited by ongoing and pre-existing shortages in health-care equipment (medications and disposable equipment) as a result of the ongoing Israeli military occupation (Moss \& Majadle, 2020). Moreover, it was found that health care workers in the occupied Palestinian territory are underprepared, lacking adequate personal protective equipment, and the lack of local protocols and training has left them with exceedingly low confidence (Alser et al., 2020). Additional elements that may
COVID-19 stress, mental distress, resilience, and well-being 
Guido Veronese, Fayez Mahamid, Dana Bdier, Rachel Pancake make the situation worse are the critical economic situation, the water and electricity crisis, poverty, and high population density (Abed, 2020).

In a population where resources of resiliency are already depleted by years of military occupation, economic crisis, health emergency, and political uncertainty, COVID-19 could be considered a new critical occurrence demanding and depleting the people's resiliency recourses. Simultaneously, subjective wellbeing and quality of life are affected by the deterioration of living conditions during the pandemic. Fear of becoming infected and a shortage of foreign aid increase the sense of isolation and threat in a territory where human and civil rights are limited due to decades of colonial domination and systematic violence (Muhareb \& Giacaman, 2020).

Our conceptual model, although preliminary in nature, can indicate the pivotal role of SWB and resilience in contexts where the two constructs are severely at risk due to environmental and political constrains (Muhareb \& Giacaman, 2020). When combining the sensitive psychological consequences of being locked down or socially distanced in a society where source of resilience and subjective well-being are conserved (Yıldırım \& Arslan, 2020), with a context characterized by poor resources for individual and collective well-being, the risk of developing severe psychological burdens and negative mental health outcomes could dramatically increase among the general population (Giacaman et al., 2011).

\section{LIMITATIONS}

Some limitations of this study must be acknowledged and discussed. This is a cross-sectional research design built on purposive sampling procedures that may have affected the generalizability of the results. However, anecdotal, and clinical experience demonstrated the precarious equilibrium of the Palestinian resilience and resistance, confirming that crucial protective factors could be at risk due to the spread of the virus in Palestine. Our findings are exploratory by nature and it is recommended that future research be extended to longitudinal designs and large-scale studies to better explain the associations between stress of COVID-19 and mental health, as well as the mediating role of resilience and subjective well-being.

A certain degree of collinearity between variables could have affected our findings, mainly regarding an overlap between the COVID-19 Stress Scales dimensions and the stress factor of the DASS. However, the COVID-19 Stress Scales have been used to detect nonclinical reactions to the pandemic outbreak (Cortez et al., 2020). On the other hand, the stress dimension of the DASS measure has been widely used for early detection of psychiatric disorders (Wang et al., 2020a, b; Zandifar et al., 2020). As for the purpose of our re- search, the association between the DASS stress factor in its clinical relevance and COVID-19 stress seems to have helped in identifying stress-related clinical outcomes due to COVID-19-related stressors. Thus, we can imagine a convergent validity between the two scales rather than redundancy of the measures.

The sample selection could have excluded portions of the population and important variables, such as socio-economic position, educational level and employment status, from analysis due to limitations in access to technology, restricting exposure to online surveys. We assume that those people could be more exposed, both to the consequences of COVID-19 and the hardships related to the military occupation. An investigation of COVID-19 consequences on the most vulnerable populations in the West Bank is strongly recommended. Furthermore, qualitative, and ethnographic research would foster a better understanding of the relationship between COVID-19 stress and its effect on the protective factors contributing to the survival of Palestinians.

Future research must also address the gender imbalance of the present pilot study; though its direction is promising, the results are not generalizable. Thus, the present work is to be considered preliminary and exploratory in its nature as it is not able to show causal relationships between the variables and did not include important socio-demographic indicators that characterize vulnerable groups.

Finally, clinical work must be oriented at promoting community and individual resilience among the most vulnerable populations in the Occupied Palestinian Territory and globally. Considering the social and political suffering of the people living in the West Bank, psychosocial and systemic interventions aimed at intervening at the community level, rather than exclusively at the individual, micro levels, are highly recommended (WHO, 2019).

Ultimately, strengthening community mental health services in a territory characterized by extreme political and economic instability is a priority (PeConga et al., 2020). If Palestinians are not guaranteed their well-being and quality of life, their endurance and resistance may be severely undermined.

\section{CONCLUSIONS}

Mental health and psychological well-being are at risk because of the COVID-19 outbreak worldwide (Holmes et al., 2020; Tandon, 2020). In Palestine, underfunded services and inadequate policies on mental health can compromise individual and collective resilience, subjective well-being, and mental health. During the pandemic, a relevant extent of the population in Palestine experienced more insecurity and uncertainty (Hammoudeh et al., 2020) under the additional stresses of COVID-19. 
The current pandemic is exposing the overall collapse of the Palestinian quality of life and poses an even greater risk to the entire population's mental health and security (Bosqui, 2020). Our findings illustrate the urgent need for immediate action to improve living conditions for the Palestinians to fortify their capacity to resist, endure and restore resilience and subjective well-being in the context of military occupation and political violence (Moss \& Majadle, 2020) during and after the pandemic. As stated by the Geneva Convention, the international community should ask the occupying forces to guarantee the occupied population the basic health, social, economic, and environmental resources that would help the population in conserving and mobilizing sources of resiliency and well-being. Otherwise, undermining the quality of life of an entire population would expose them to a high risk of social and individual suffering, mainly during a global crisis such as that of COVID-19 (AlKhaldi et al., 2020).

\section{ReFERENCES}

Agbaria, Q., \& Natur, N. (2018). The relationship between violence in the family and adolescent's aggression: The mediator role of self-control, social support, religiosity, and well-being. Children and Youth Services Review, 91, 447-456. https://doi.org/ 10.1016/j.childyouth.2018.06.016

Abed, Y. (2020). COVID-19 in the Gaza Strip and the West Bank under the political conflict in Palestine. South Eastern European Journal of Public Health (SEEJPH). https://doi.org/10.4119/seejph-3543

Ahmed, M. Z., Ahmed, O., Aibao, Z., Hanbin, S., Siyu, L., \& Ahmad, A. (2020). Epidemic of COVID-19 in China and associated psychological problems. Asian Journal of Psychiatry, 51, 102092. https://doi. org/10.1016/j.ajp.2020.102092

Ahuja, K. K., Banerjee, D., Chaudhary, K., \& Gidwani, C. (2020). Fear, xenophobia, and collectivism as predictors of well-being during coronavirus disease 2019: an empirical study from India. International Journal of Social Psychiatry. https:// doi.org/10.1177/0020764020936323

Aldwin, C. M. (2007). Stress, coping, and development: an integrative perspective. Guilford Press.

AlKhaldi, M., Kaloti, R., Shella, D., Al Basuoni, A., \& Meghari, H. (2020). Health system's response to the COVID-19 pandemic in conflict settings: Policy reflections from Palestine. Global Public Health, 15, 1244-1256. https://doi.org/10.1080/17441692.20 20.1781914

Alser, O., Alghoul, H., Alkhateeb, Z., Hamdan, A., Albaraqouni, L., \& Saini, K. (2020). Healthcare workers preparedness for COVID-19 pandemic in the occupied Palestinian territory: a cross-sectional survey. MedRxiv. https://doi.org/10.1101/2020.05.09.20096099
Arslan, G., \& Yildirim, M. (2020a). Meaningful living, resilience, affective balance, and psychological health problems during COVID-19. PsyArXiv. https://doi.org/10.31234/osf.io/wsr3e

Arslan, G., \& Yıldırım, M. (2020b). Coronavirus stress, meaningful living, optimism, and depressive symptoms: a study of moderated mediation model. PsyArXiv. https://doi.org/10.31234/osf.io/ykvzn

Asi, Y. M., Unruh, L., \& Liu, X. (2018). Conflict and well-being: a comparative study of health-related quality of life, stress, and insecurity of university students in the West Bank and Jordan. Quality of Life Research, 27, 1381-1391. https://doi.org/ 10.1007/s11136-018-1802-y

Bosqui, T. (2020). The need to shift to a contextualized and collective mental health paradigm: Learning from crisis-hit Lebanon. Global Mental Health, 7, e26. https://doi.org/10.1017/gmh.2020.20

Burtscher, J., Burtscher, M., \& Millet, G. P. (2020). (Indoor) isolation, stress, and physical inactivity: Vicious circles accelerated by COVID-19? Scandinavian Journal of Medicine \& Science in Sports, 30, 1544-1545. https://doi.org/10.1111/sms.13706

Cortez, P. A., Joseph, S. J., Das, N., Bhandari, S. S., \& Shoib, S. (2020). Tools to measure psychological impact of COVID-19 pandemic: What do we have in the platter? Asian Journal of Psychiatry, 53, 102371. https://doi.org/10.1016/j.ajp.2020.102371

Dashraath, P., Jeslyn, W. J. L., Karen, L. M. X., Min, L. L., Sarah, L., Biswas, A., Choolani, M., Mattar, C., \& Su, L. L. (2020). Coronavirus disease 2019 (COVID-19) pandemic and pregnancy. American Journal of Obstetrics and Gynecology, 222, 521-531. https://doi.org/10.1016/j.ajog.2020.03.021

Dong, M., \& Zheng, J. (2020). Letter to the editor: Headline stress disorder caused by netnews during the outbreak of COVID-19. Health Expectations: An International Journal of Public Participation in Health Care and Health Policy, 23, 259-260. https://doi.org/10.1111/hex.13055

Freire, C., Ferradás, M. D. M., Valle, A., Núñez, J. C., \& Vallejo, G. (2016). Profiles of psychological wellbeing and coping strategies among university students. Frontiers in Psychology, 7, 1554. https://doi. org/10.3389/fpsyg.2016.01554

Friborg, O., Hjemdal, O., Rosenvinge, J. H., \& Martinussen, M. (2003). A new rating scale for adult resilience: What are the central protective resources behind healthy adjustment? International Journal of Methods in Psychiatric Research 12, 65-76. https://doi.org/10.1002/mpr.143

Gao, J., Tian, Z., \& Yang, X. (2020). Breakthrough: Chloroquine phosphate has shown apparent efficacy in treatment of COVID-19 associated pneumonia in clinical studies. Bioscience Trends, 14, 72-73. https://doi.org/10.5582/bst.2020.01047

Giacaman, R., Rabaia, Y., Nguyen-Gillham, V., Batniji, R., Punamäki, R. L., \& Summerfield, D. (2011).
COVID-19 stress, mental distress, resilience, and well-being 
Guido Veronese, Fayez Mahamid, Dana Bdier, Rachel Pancake
Mental health, social distress and political oppression: The case of the occupied Palestinian territory. Global Public Health, 6, 547-559. https:// doi.org/10.1080/17441692.2010.528443

Ghandour, R., Ghanayem, R., Alkhanafsa, F., Alsharif, A., Asfour, H., Hoshiya, A., Masalmeh, A., Nadi, M., Othman, L., Ryahe, S., Wahdan, Y., Wahsh, S., Yamani, A., \& Giacaman, R. (2020). Double burden of COVID-19 pandemic and military occupation: Mental health among a Palestinian university community in the West Bank. Annals of Global Health, 86, 131. https://doi.org/10.5334/ aogh.3007

Gunzler, D., Chen, T., Wu, P., \& Zhang, H. (2013). Introduction to mediation analysis with structural equation modeling. Shanghai Archives of Psychiatry, 25, 390-394. https://doi.org/10.3969/j.issn.10020829.2013.06.009

Hair, J. F., Black, W. C., Babin, B. J., \& Anderson, R. E. (2010). Multivariate data analysis. Pearson Education Inc.

Hambleton, R. K., Merenda, P. F., \& Spielberger, C. D. (Eds.) (2004). Adapting educational and psychological tests for cross-cultural assessment. Psychology Press.

Hammoudeh, W., Jabr, S., Helbich, M., \& Sousa, C. (2020). On mental health amid COVID-19. Journal of Palestine Studies, 49, 77-90. https://doi. org/10.1525/jps.2020.49.4.77

Holmes, E. A., O'Connor, R. C., Perry, V. H., Tracey, I., Wessely, S., Arseneault, L., Ballard, C., Christensen, H., Cohen Silver, R., Everall, I., Ford, T., John, A., Kabir, T., King, K., Madan, I., Michie, S., Przybylski, A. K., Shafran, R., Sweeney, A., ... Bullmore, E. (2020). Multidisciplinary research priorities for the COVID-19 pandemic: a call for action for mental health science. The Lancet Psychiatry, 7, 547-560. https://doi.org/10.1016/S22150366(20)30168-1

Hu, L., \& Bentler, P. M. (1999). Cutoff criteria for fit indexes in covariance structure analysis: Conventional criteria versus new alternatives. Structural Equation Modeling, 6, 1-55. https://doi.org/ 10.1080/10705519909540118

Islam, S. D. U., Bodrud-Doza, M., Khan, R. M., Haque, M. A., \& Mamun, M. A. (2020). Exploring COVID-19 stress and its factors in Bangladesh: a perception-based study. Heliyon, 6, e04399. https://doi.org/10.1016/j.heliyon.2020.e04399

Kavčič, T., Avsec, A., \& Kocjan, G. Z. (2021). Psychological functioning of Slovene adults during the COVID-19 pandemic: Does resilience matter? Psychiatric Quarterly, 92, 207-216. https://doi. org/10.1007/s11126-020-09789-4

Kteily-Hawa, R., Khalifa, D. S., \& Abuelaish, I. (2020). Resilience among a large sample of adult Palestinians in the Gaza Strip: Examining contextual sociodemographic factors and emotional response through a social-ecological lens. Public Health, 182, 139-142. https://doi.org/10.1016/j.puhe.2019.12.004

Layous, K., Chancellor, J., \& Lyubomirsky, S. (2014). Positive activities as protective factors against mental health conditions. Journal of Abnormal Psychology, 123, 3-12. https://doi.org/10.1037/a0034709

Liu, C. H., Zhang, E., Wong, G. T. F., \& Hyun, S. (2020). Factors associated with depression, anxiety, and PTSD symptomatology during the COVID-19 pandemic: Clinical implications for US young adult mental health. Psychiatry Research, 290, 113172. https://doi.org/10.1016/j.psychres.2020.113172

Lovibond, P. F., \& Lovibond, S. H. (1995). The structure of negative emotional states: Comparison of the Depression Anxiety Stress Scales (DASS) with the Beck Depression and Anxiety Inventories. Behaviour Research and Therapy, 33, 335-343. https:// doi.org/10.1016/0005-7967(94)00075-u

Mahamid, F. A., \& Berte, D. Z. (2019). Social media addiction in geopolitically at-risk youth. International Journal of Mental Health and Addiction, 17, 102-111. https://doi.org/10.1007/s11469-017-9870-8 Mahamid, F. A., \& Berte, D. Z. (2020). Portrayals of violence and at-risk populations: Symptoms of trauma in adolescents with high utilization of social media. International Journal of Mental Health and Addiction, 18, 980-992. https://doi.org/10.1007/ s11469-018-9999-0

Mahamid, F., \& Bdier, D. (2020a). The association between positive religious coping, perceived stress, and depressive symptoms during the spread of coronavirus (COVID-19) among a sample of adults in Palestine: a cross-sectional study. Journal of Religion and Health, 60, 34-49. https://doi. org/10.1007/s10943-020-01121-5

Mahamid, F., \& Bdier, D. (2020b). Fear of COVID-19 and mental health outcomes among psychosocial service providers in Palestine: The mediating role of wellbeing. Retrieved from https://concurrentdisorders. ca/2020/12/24/fear-of-covid-19-and-mental-healthoutcomes-among-psychosocial-service-providersin-palestine-the-mediating-role-of-well-being

Mahamid, F., Bdier, D., \& Berte, D. Z. (2020a). Psychometric properties of the Fear of COVID-19 Scale (FCV-19S) in a Palestinian context. Manuscript submitted for publication.

Mahamid, F., Veronese, G., \& Bdier, D. (2020b). Fear of coronavirus COVID-19 and mental health outcomes in Palestine: The mediating role of social support. Manuscript submitted for publication.

Marsh, H. W., Hau, K. T., \& Wen, Z. (2004). In search of golden rules: Comment on hypothesis-testing approaches to setting cutoff values for fit indexes and dangers in overgeneralizing Hu and Bentler's (1999) findings. Structural Equation Modeling, 11, 320-341. https://doi.org/10.1207/s15328007sem1103_2

Montano, R. L. T., \& Acebes, K. M. L. (2020). COVID stress predicts depression, anxiety, and stress 
symptoms of Filipino respondents. International Journal of Research in Business and Social Science, 9, 78-103. https://doi.org/10.20525/ijrbs.v9i4.773

Moss, D., \& Majadle, G. (2020). Battling COVID-19 in the occupied Palestinian territory. The Lancet Global Health, 8, e1127-e1128. https://doi.org/10.1016/ S2214-109X(20)30237-0

Muhareb, R., \& Giacaman, R. (2020). Tracking COVID-19 responsibly. The Lancet. https://doi. org/10.1016/S0140-6736(20)30693-0

PeConga, E. K., Gauthier, G. M., Holloway, A., Walker, R. S., Rosencrans, P. L., Zoellner, L. A., \& Bedard-Gilligan, M. (2020). Resilience is spreading: Mental health within the COVID-19 pandemic. Psychological Trauma: Theory, Research, Practice, and Policy, 12, S47-S48. https://doi.org/10.1037/ tra0000874

Pfefferbaum, B., \& North, C. S. (2020). Mental health and the COVID-19 pandemic. New England Journal of Medicine, 383, 510-512. https://doi.org/10.1056/ NEJMp2008017

Poyiadji, N., Shahin, G., Noujaim, D., Stone, M., Patel, S., \& Griffith, B. (2020). COVID-19-associated acute hemorrhagic necrotizing encephalopathy: CT and MRI features. Radiology, 296, 201187. https://doi.org/10.1148/radiol.2020201187

Schermelleh-Engel, K., Moosbrugger, H., \& Müller, H. (2003). Evaluating the fit of structural equation models: Tests of significance and descriptive goodness-of-fit measures. Methods of Psychological Research Online, 8, 23-74.

Spinelli, A., \& Pellino, G. (2020). COVID-19 pandemic: Perspectives on an unfolding crisis. The British Journal of Surgery, 107, 785-787. https://doi. org/10.1002/bjs. 11627

Staehr, J. K. (1998). The use of well-being measures in primary health care - the DepCare project. In WHO Regional Office for Europe (Ed.), Well-being measures in primary health care - the DepCare project. WHO.

Stevenson, C., \& Wakefield, J. (2020, July 14). Financial distress and suicidal behaviour during COVID-19: Family identification attenuates the negative relationship between financial distress and mental ill-health. OSF Preprints. https://doi.org/10.31219/ osf.io/yuqzm

Tandon R. (2020). COVID-19 and mental health: Preserving humanity, maintaining sanity, and promoting health. Asian Journal of Psychiatry, 51, 102256. https://doi.org/10.1016/j.ajp.2020.102256

Taylor, S., Landry, C., Paluszek, M., Fergus, T. A., McKay, D., \& Asmundson, G. J. (2020). Development and initial validation of the COVID Stress Scales. Journal of Anxiety Disorders, 72, 102232. https://doi.org/10.1016/j.janxdis.2020.102232

Torales, J., O’Higgins, M., Castaldelli-Maia, J. M., \& Ventriglio, A. (2020). The outbreak of COVID-19 coronavirus and its impact on global mental health.
International Journal of Social Psychiatry, 66, 317320. https://doi.org/10.1177/0020764020915212

Trzebiński, J., Cabański, M., \& Czarnecka, J. Z. (2020). Reaction to the COVID-19 pandemic: The influence of meaning in life, life satisfaction, and assumptions on world orderliness and positivity. Journal of Loss and Trauma, 25, 544-557. https:// doi.org/10.1080/15325024.2020.1765098

Veronese, G., Pepe, A., Jaradah, A., Al Muranak, F., \& Hamdouna, H. (2017). Modelling life satisfaction and adjustment to trauma in children exposed to ongoing military violence: An exploratory study in Palestine. Child Abuse \& Neglect, 63, 61-72. https://doi.org/10.1016/j.chiabu.2016.11.018

Veronese, G., Pepe, A., Alzaanin, W., \& Shoman, H. (2020a). Sources of functioning, symptoms of trauma, and psychological distress: a cross-sectional study with Palestinian health workers operating in West Bank and Gaza Strip. American Journal of Orthopsychiatry, 90, 751-759. https://doi.org/10.1037/ ort0000508

Veronese, G., Sousa, C., \& Cavazzoni, F. (2020b). Survival and resilience among Palestinian women: a qualitative analysis using individual and collective life events calendars. Violence Against Women. https://doi.org/10.1177/1077801220914406

Wang, C., Pan, R., Wan, X., Tan, Y., Xu, L., Ho, C. S., \& Ho, R. C. (2020a). Immediate psychological responses and associated factors during the initial stage of the 2019 coronavirus disease (COVID-19) epidemic among the general population in China. International Journal of Environmental Research and Public Health, 17, 1729. https://doi.org/10.3390/ ijerph17051729

Wang, C., Pan, R., Wan, X., Tan, Y., Xu, L., McIntyre, R. S., Choo, F. N., Tran, B., Ho, R., Sharma, V. K., \& Ho, C. (2020b). A longitudinal study on the mental health of general population during the COVID-19 epidemic in China. Brain, Behavior, and Immunity, 87, 40-48. https://doi.org/10.1016/j. bbi.2020.04.028

World Health Organization (2001) Mental health: New understanding, new hope. The world health report. WHO.

World Health Organization (2019). Coronavirus disease 2019 (COVID-19): Situation report. WHO.

World Health Organization (2020a). WHO coronavirus disease (COVID-19) dashboard. Retrieved from https://covid 19.who.int/ [accessed July 26, 2020]

World Health Organization (2020b). WHO coronavirus disease (COVID-19) dashboard. Occupied Palestinian territory, including east Jerusalem. Retrieved from https://covid19.who.int/region/emro/country/ ps [accessed July 26, 2020]

Xiao, H., Zhang, Y., Kong, D., Li, S., \& Yang, N. (2020). Social capital and sleep quality in individuals who self-isolated for 14 days during the coronavirus disease 2019 (COVID-19) outbreak in Janu-
COVID-19 stress, mental distress, resilience, and well-being 
Guido Veronese, Fayez Mahamid, Dana Bdier, Rachel Pancake ary 2020 in China. Medical Science Monitor, 26, e923921. https://doi.org/10.12659/MSM.923921

Yang, H., \& Ma, J. (2020). How an epidemic outbreak impacts happiness: Factors that worsen (vs. protect) emotional well-being during the coronavirus pandemic. Psychiatry Research, 289, 113045. https://doi.org/10.1016/j.psychres.2020.113045

Yıldırım, M., \& Arslan, G. (2020). Exploring the associations between resilience, dispositional hope, preventive behaviours, subjective well-being, and psychological health among adults during early stage of COVID-19. Current Psychology. https:// doi.org/10.1007/s12144-020-01177-2

Zandifar, A., Badrfam, R., Yazdani, S., Arzaghi, S. M., Rahimi, F., Ghasemi, S., Khamisabadi, S., Mohammadian Khonsari, N., \& Qorbani, M. (2020). Prevalence and severity of depression, anxiety, stress and perceived stress in hospitalized patients with COVID-19. Journal of Diabetes and Metabolic Disorders, 19, 1-8. https://doi.org/10.1007/s40200-02000667-1

Zolkoski, S. M., \& Bullock, L. M. (2012). Resilience in children and youth: a review. Children and Youth Services Review, 34, 2295-2303. https://doi. org/10.1016/j.childyouth.2012.08.009 